NEUROSCIENCE

\section{Alzheimer's clue from spatial test}

Young adults who are at increased risk of developing Alzheimer's disease show abnormal function in a part of the brain involved in spatial navigation.

Nikolai Axmacher at Ruhr University Bochum in Germany and his colleagues used neuroimaging to measure the functioning of the 'gridcell' system in the entorhinal cortex as participants with or without the $A P O E-\varepsilon 4$ risk gene navigated a virtual arena.

The 38 high-risk individuals showed reduced grid-cell functioning compared with the 37 controls and tended to avoid the centre of the arena. Activity in the hippocampal region was higher when the grid-cell system was impaired, as if to compensate for the deficit. Reduced grid-cell functioning may prove useful as an early biomarker of Alzheimer's, the authors suggest.

Science 350, 430-433 (2015)

\section{ASTRONOMY}

\section{Red-giant rogue in Andromeda}

Astronomers have spotted a giant 'runaway star' speeding through the Andromeda galaxy; the first of its kind seen outside the Milky Way.

Whereas most stars flow together around the centre of their galaxy, some, known as runaways, travel at different directions and speeds to their peers, and may even escape their galaxy entirely. Spotting red-supergiant runaways is rare - stars in this late stage of life left their birthplace long ago, making their unusual speeds harder to spot than for younger runaways.

Kate Evans and Philip

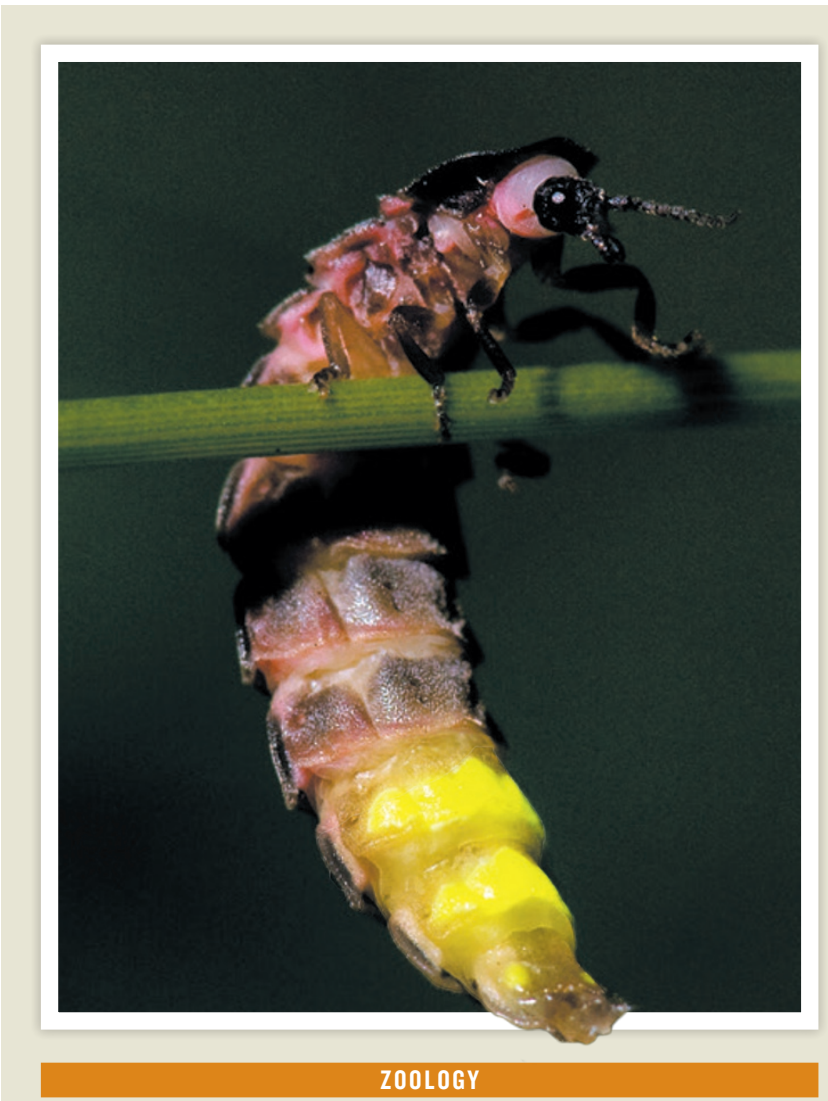

\section{Bright light as sex signal}

Brighter female glow-worms lay more eggs than their dim rivals and are more attractive to potential nocturnal mates.

Juhani Hopkins at the University of Oulu in Finland and his colleagues allowed 26 female glow-worms (Lampyris noctiluca; pictured) to mate in the lab. The glowing lanterns of the insects varied in size from 7 square millimetres to 19 square millimetres - larger lanterns produce a brighter glow. Each glow-worm laid between 25 and 195 eggs, with those perceived by the researchers to be brightest laying the most. Male glow-worms presented with fake females also preferred those with brighter lights.

The lanterns of female glow-worms may provide clues about fitness to males, who are unable to assess size - also an indicator of fecundity - in the dark.

Biol. Lett. http://dx.doi.org/10.1098/rsbl.2015.0599 (2015)
Massey at the Lowell Observatory in Flagstaff, Arizona, studied a red supergiant in the Andromeda galaxy known as J004330.06+405258.4 and calculated that it is travelling 400-450 kilometres per second faster than its neighbours. The star is the first massive runaway to be spotted outside our own galaxy and the fastest anywhere for its size, say the authors.

Astron. J. 150, 149 (2015)

\section{MATERIALS}

\section{Iron skin senses the softest touch}

An iron-based artificial skin can sense the lightest touch.

Ahmed Alfadhel and Jürgen Kosel at the King Abdullah University of Science and Technology in Thuwal, Saudi Arabia, made a tactile sensor by embedding iron nanowires in hair-like structures called cilia, made of a polymer called polydimethylsiloxane, on a magnetic sensor. When the magnetized cilia are bent by touch, they trigger the sensor.

The skin can flex and, depending on the size of the cilia, can be sensitive enough to measure a person's wrist pulse. Because the cilia use permanent magnets rather than electromagnets, the device does not require much power and - unlike most other tactile sensors - it can work underwater and measure liquid flow, the authors say. Adv. Mater. http://doi.org/f3jp2n (2015)

\section{GENOMICS}

\section{Gene regulation predates animals}

The oldest ancestor of animal life used the same tricks that modern humans do to turn genes on and off.

Alex de Mendoza at the Institute of Evolutionary Biology in Barcelona, Spain, and his colleagues studied gene regulation in the funguslike single-celled organism Creolimax fragrantissima, which branched onto a separate evolutionary path before the evolution of multicellular organisms.

To produce different cell types, multicellular organisms use three main gene-regulation processes: transcription factors, alternative splicing and 